\title{
Combined Search for UHE Neutrinos from Binary Black Hole Mergers with the Pierre Auger Observatory
}

\author{
Michael Schimp ${ }^{a * *}$ on behalf of the Pierre Auger $^{b}$ Collaboration \\ (a complete list of authors can be found at the end of the proceedings) \\ ${ }^{a}$ Bergische Universität Wuppertal, Department of Physics, Wuppertal, Germany \\ ${ }^{b}$ Observatorio Pierre Auger, Av. San Martín Norte 304, 5613 Malargüe, Argentina \\ E-mail: spokespersons@auger.org
}

\begin{abstract}
We present searches for ultra-high energy (UHE) neutrinos ( $>0.1 \mathrm{EeV}$ ) with the Pierre Auger Observatory, following up binary black hole (BBH) mergers detected by the LIGO and Virgo detectors via gravitational waves (GWs). In this work, the so-far published BBH mergers are combined as standard candles with a hypothetical isotropic UHE neutrino luminosity $L\left(t-t_{0}\right)$ as a function of the time after the respective merger, $t-t_{0}$. The UHE neutrino emission spectrum is assumed to follow a power law distribution $\propto E_{v}^{-2}$. Using these assumptions, $L\left(t-t_{0}\right)$ is probed, taking into account the instantaneous effective area of the Pierre Auger Observatory to UHE neutrinos and the 3D sky localizations of the sources. No UHE neutrino candidates have been found and upper limits on $L\left(t-t_{0}\right)$ are obtained for the hypothetical cases of emissions lasting 24 hours and 60 days after the merger, respectively. The corresponding upper limit on the total energy per source emitted in UHE neutrinos does not depend on the emission duration and demonstrates the competitiveness of the Pierre Auger Observatory with dedicated neutrino telescopes.
\end{abstract}

$37^{\text {th }}$ International Cosmic Ray Conference (ICRC 2021)

July 12th - 23rd, 2021

Online - Berlin, Germany

\footnotetext{
*Presenter
} 


\section{Introduction}

The Pierre Auger Observatory near Malargüe in the Province of Mendoza, Argentina, is the largest and most precise UHE cosmic ray (UHECR) detector in the world. The Observatory consists of several detection systems, one of which is the surface detector (SD), a triangular grid consisting of 1660 water-Cherenkov detectors (WCDs) with a $1.5 \mathrm{~km}$ spacing. Overall, the SD covers an area of $3000 \mathrm{~km}^{2}$ at an average altitude of $1400 \mathrm{~m}$ above sea level, corresponding to a vertical atmospheric depth of $875 \mathrm{~g} / \mathrm{cm}^{2}$. Each WCD is filled with 12 tons of ultra-pure water. Charged particles from extensive air showers (EASs) cause the emission of Cherenkov radiation in the water, which is detected using photomultiplier tubes (PMTs). The PMT signals are used to determine EAS properties such as the arrival direction, energy, and identity of the primary particle. The Pierre Auger Observatory has been taking data since the beginning of 2004. Its components are described in detail in [1].

Searches for steady fluxes - both diffuse and direction-dependent - of UHE neutrinos, carrying energies beyond $10^{17} \mathrm{eV}$, with the Pierre Auger Observatory have been successfully performed [2, 3], substantially constraining several source scenarios. These searches rely on the distinguishability of inclined EASs induced by UHECRs from those induced by UHE neutrinos, based on the difference in the shower particle compositions reaching the ground, which are associated with different signal shapes as a function of time in the PMTs. UHE neutrinos are thought to originate from UHECRs, being produced either at UHECR sources or during their propagation through the Universe [4-8]. As neutrinos are electrically neutral, they are not deflected in magnetic fields, and their arrival directions thus point back to their origins. In addition, neutrinos are only subject to the weak interaction, such that they can reach Earth from virtually arbitrary distances due to the lack of attenuation. In conclusion, neutrinos are a suitable component in multi-messenger campaigns in combination with any other astrophysical messenger particle.

The LIGO Scientific Collaboration discovered BBH mergers with the very first directly detected GWs in 2015, initiating the era of GW astronomy [9, 10]. Starting with this discovery, the LIGO Scientific Collaboration and Virgo Collaboration (LVC) have so far reported the detection of GWs from $62 \mathrm{BBH}$ mergers. Searches for counterpart signals via other messengers have yielded coincident detections for some events [11-13], none of which have been sufficiently significant to claim a discovery. We present an application of UHE neutrino searches with Auger following up these BBH merger GW events. Finding no UHE neutrinos in coincidence, we constrain the emission of UHE neutrinos by BBH mergers with a simple isotropic standard candle hypothesis.

This work is an enhancement of a previously presented combined search that used only the first 21 published GW events from BBH mergers [14]. Besides the extended set of sources, we improved the method by taking into account the full provided 3D sky localization probability density function (PDF) and added a 60-day follow-up search period to the already established period of 24 hours in order to probe a potential long-term emission.

In the following sections, the follow-up search methods, parameters, and results are discussed.

\section{LIGO/Virgo Binary Black Hole Merger Event Information}

The 62 BBH merger events followed up for this work originate from three different sets: 
1. The GW event catalogue GWTC-1 [15], containing 11 high-confidence GW events from the LIGO/Virgo runs $\mathrm{O} 1$ and $\mathrm{O} 2,10$ of which are most likely from $\mathrm{BBH}$ mergers. The other event is the binary neutron star merger GW170817, which is not considered in this work but has been followed up separately [16].

2. The GW event catalogue GWTC-2 [17], containing high-confidence GW events from the LIGO/Virgo run O3 until the end of September 2019. This part of O3 is also called O3a. In accordance to [17], the 36 events with the lighter object's mass $m_{2}>3 M_{\odot}$ in this catalogue are taken as BBH mergers. The GWTC-2 data release contains different parameter estimations for each source, including a suitable weighted average, which is used for this work.

3. The 18 high-confidence $\mathrm{BBH}$ merger events of the remaining part of LIGO/Virgo run O3, called $\mathrm{O} 3 \mathrm{~b}$ and published via public alerts [18-33]. High confidence of a BBH merger is assumed for each event with an associated probability $p_{\mathrm{BBH}}>0.75$ to originate from a BBH merger. Considering all $\mathrm{GW}$ events, it turns out $p_{\mathrm{BBH}}$ is either larger than 0.88 or very close to zero, so the analysis is not sensitive to small variations of this quantity. All events that have been retracted or found to be most likely of terrestrial origin are excluded from the analysis.

For each BBH merger event, the time of the merger $t_{0}$ as well as the 3D sky localization PDF has been provided by LVC. The directional part of the sky localization PDF is given in terms of discrete probabilities $P_{p, s}$ for each source $s$ to be located in the direction of a healpix pixel $p$ [34]. The distance part of the 3D localization PDF is given in terms of conditional probability distributions $\Pi_{p, s}(r)$, with the luminosity distance parameter $r$, provided for each source $s$ in each healpix pixel $p$. It is parametrized as a normal distribution times $r^{2}$. The overall 3D localization PDF is the product of these two parts, such that the probability of any source $s$ to be located in a spherical coordinate bin defined by a healpix pixel $p$ and a distance bin $[\rho, \rho+\Delta \rho]$ is $P_{p, s} \cdot \int_{\rho}^{\rho+\Delta \rho} \Pi_{p, s}(r) \mathrm{d} r$.

\section{Ultra-high Energy Neutrino Sensitivity of the Pierre Auger Observatory}

To quantify the direction-dependent sensitivity to UHE neutrinos, as discussed in [3], the effective area of the SD for UHE neutrino detection $A_{\mathrm{eff}}\left(E_{\nu}, \theta, t-t_{0}\right)$ as a function of the local zenith angle $\theta$ and neutrino energy $E_{v}$ is used. It reflects the geometrical area of the SD and the efficiency of the neutrino detection and identification. Its explicit time dependence originates from minor short-term changes in the status of the SD, that are applied implicitly in the final calculation.

We define $\mathcal{A}(\theta, t)=\int_{0}^{\infty} E_{\nu}^{-2} A_{\text {eff }}\left(E_{v}, \theta, t\right) \mathrm{d} E_{\nu}$ as the effective area folded with an $E_{v}^{-2}$ neutrino spectrum, which is a common assumption for UHE neutrino searches. Figure 1 shows the average of $\mathcal{A}(\theta, t)$ for the year 2016, the benchmark time period for effective areas used in [3]. Variations of $\mathcal{A}$ with $\theta$ are notable and exhibit a strong peak at $\theta=90.8^{\circ}$. Note that the healpix pixels provided by LVC are substantially smaller than the expected angular resolution for reconstructed neutrinos. This implies that the discretization of $\mathcal{A}(\theta, t)$ to $\mathcal{A}_{p}(t)$, using that $\theta$ is a function of $p$ and $t$, does not affect the precision of this analysis. 


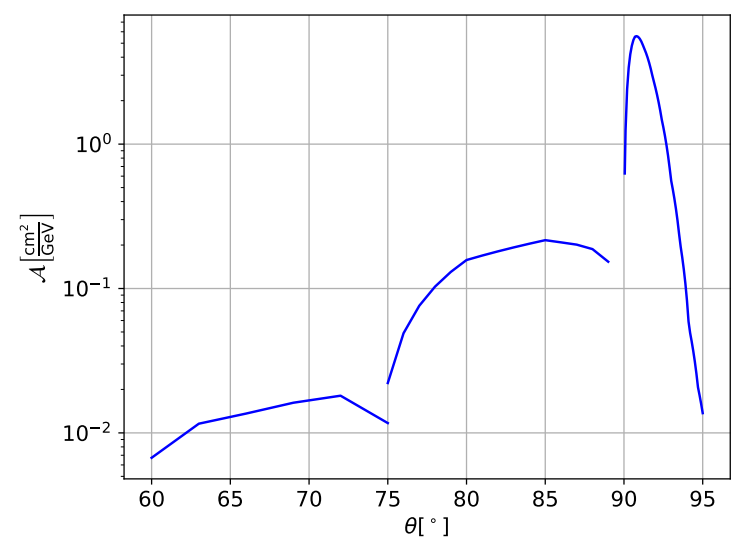

Figure 1: $\mathcal{A}(\theta, t)$ averaged over the year 2016, reflecting the $\theta$-dependent sensitivity of the SD to UHE neutrinos

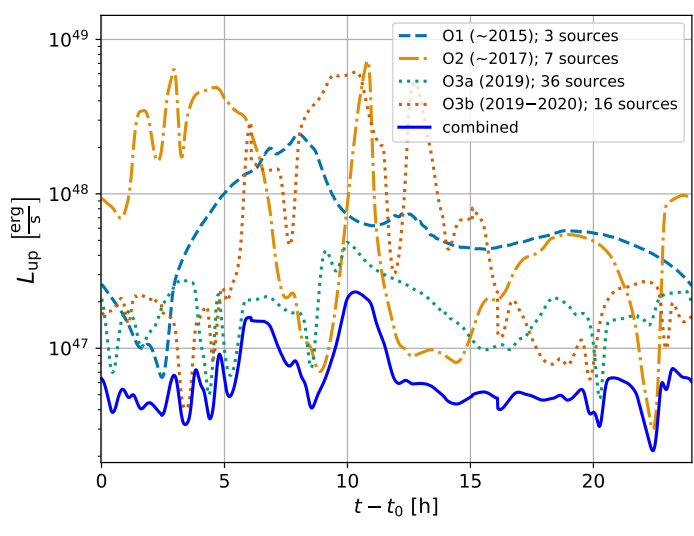

Figure 2: Solid line: upper limit (90\% C.L.) on the time-dependent universal isotropic neutrino luminosity for the 24 hour follow-up period; other lines: contributions from individual LIGO/Virgo observational runs

\section{Observation}

We applied the established neutrino searches [2] in the most probable $90 \%$ quantile of each source's directional localization PDF. The searches have been performed using two periods starting at the time of the merger $t_{0}$, namely 24 hours (in accordance with [14]), and 60 days to allow for potential longer-term emission scenarios. No neutrino candidates have been found in coincidence with any of the considered sources in any of the described search periods and directions.

\section{Source stacking}

The UHE neutrino emission from the BBH mergers detected by LVC can be constrained by combining the expected neutrino fluxes from all of them, following the approach from [14]. We impose no a priori constraints on the hypothetical isotropic UHE neutrino luminosity of the sources as a function of time after the merger, $L\left(t-t_{0}\right)$, which we assume to be the same function for each source. Using the assumption of a neutrino spectrum $\propto E_{v}^{-2}$ from each source and considering the non-observation of UHE neutrino candidates, we constrain $L\left(t-t_{0}\right)$ as follows.

The total number of identified neutrinos $N_{v}$ from the considered sources $s$ as a function of the universal isotropic neutrino luminosity $L$, the effective area $A_{\mathrm{eff}}$, the search duration $T$, and the BBH merger localization PDFs $P$ and $\Pi$ can be written as:

$$
N_{v}=\sum_{s} \sum_{p \in \Omega_{90}(s)} P_{p, s} \int_{0}^{\infty} \frac{\Pi_{p, s}(r)}{r^{2}} \mathrm{~d} r \int_{t_{0}}^{t_{0}+T} L(t) \int_{0}^{\infty} E_{\nu}^{-2} A_{\text {eff }, p}\left(E_{v}, t\right) \mathrm{d} E_{v} \mathrm{~d} t,
$$

where $\Omega_{90}(s)$ is the solid angle of the most probable $90 \%$ quantile of the source's directional localization PDF. The integral over $r$ yields an effective factor for the inverse distance squared considering the distance PDF $\Pi$. As the integrand is a shifted normal distribution, the result is 
evaluated using the Gauss error function. The zenith-angle dependence of $A_{\text {eff }}$ is contained in its pixel index $p$, which, at a given time, always yields a fixed zenith angle. We furthermore identify the energy integral $\int_{0}^{\infty} E_{\nu}^{-2} A_{\text {eff, } p}\left(E_{v}, t\right) \mathrm{d} E_{v}=\mathcal{A}_{p}(t)$ as in Figure 1.

The non-observation of neutrino candidates with a background low enough to be compatible with zero allows to set the $90 \%$ C.L. upper limit $N_{\text {up }, v}=2.44$ [35]. Taking the time integral as a sum over discrete 1-second-long time bins $i$ and applying no prior on the form of the isotropic UHE neutrino luminosity upper limit allows to solve Equation 1 for it:

$$
L_{\mathrm{up}, i}=\frac{N_{\mathrm{up}, v}}{T}\left(\sum_{s} \sum_{p \epsilon \Omega_{9_{0}}(s)} P_{p, s} \mathcal{A}_{p, s, i} \int_{0}^{\infty} \frac{\Pi_{p, s}(r)}{r^{2}} \mathrm{~d} r\right)^{-1} .
$$

The denominator $T$ is a product of two factors: the number of time bins, over which the upper limit of observed neutrino candidates is evenly distributed as both the number of observed candidates as well as the number of expected background events are the same at all times (zero); and the time bin width, which originates from the differential $\mathrm{d} t$ from the time integration in Equation 1.

\section{Results}

The resulting isotropic UHE neutrino source luminosity upper limit $L_{\mathrm{up}, i}$ for the 24-hour followup period is shown in Figure 2, where the contributions from the individual LVC observational runs are indicated as well. Runs that contribute predominantly to the combined sensitivity at certain times are represented by lower values for these times in Figure 2. The upper limit obtained by combining all runs is below $10^{47} \frac{\mathrm{erg}}{\mathrm{s}}$ for most of the considered period. Approximately 22.4 hours after the merger, the combined sensitivity is maximal, leading to the exclusion of a universal isotropic UHE neutrino source luminosity above approximately $2.2 \cdot 10^{46} \frac{\mathrm{erg}}{\mathrm{s}}$. The variations of the combined limit are smaller than those for single runs, as the variations in the visibilities of all sources are averaged out. As expected, the combination of sources and runs substantially improves the sensitivity to the source class of BBH mergers compared to single sources or runs. The time integral of $L_{\mathrm{up}, i}$ yields the upper limit on the total emitted energy in terms of neutrinos per source, $E_{\text {up }}$, still assuming an emission $\propto E_{v}^{-2}$. The resulting value for $E_{\text {up }}$ is approximately $6.0 \cdot 10^{51} \mathrm{erg}$.

The results for $L_{\mathrm{up}, i}$ for the 60-day follow-up period are shown in Figures 3 and 4 . Figure 3 shows the combined limit for the 60-day search period with one line representing each sidereal day. It illustrates both the daily periodicity and variations of the 60-day luminosity limit. The periodicity originates from the periodic visibility of the sources while the variations originate from the time dependence of $\mathcal{A}(t)$, which reflects minor changes in the status of the SD such as individual stations' communication systems suspending or resuming. The luminosity limit values are approximately a factor of 60 smaller than the ones for the 24-hour follow up in accordance to Equation 2, where it is shown that the limit scales with the inverse of the search period duration.

A representation of the 60-day limit analogous to Figure 2 with the same time resolution would have frequent and large oscillations of the lines, making it barely discernible. Therefore, Figure 4 shows the combined limit for the 60-day search period in terms of the (sidereal) daily mean value. It illustrates the stability of the time-dependent limit over the 60-day search period both for the overall limit as well as for the contributions of the individual runs. The combination of the runs increases 


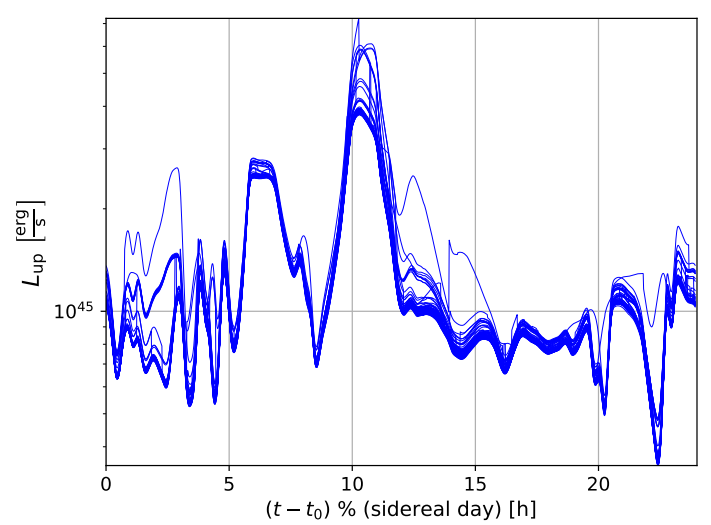

Figure 3: Sidereal daily upper limit (90\% C.L.) on the time-dependent universal isotropic neutrino luminosity for the 60 day follow-up period; lines are wrapped around to illustrate the periodicity and variations of values

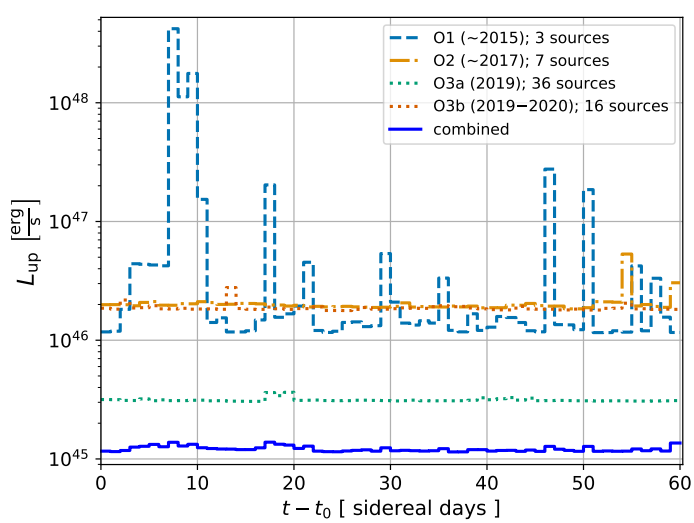

Figure 4: Solid line: sidereal daily average upper limit (90 \% C.L.) on the time-dependent universal isotropic neutrino luminosity for the 60 day followup period; other lines: contributions from individual LIGO/Virgo observational runs

the stability of the limit since a larger number of considered sources leads to more efficient averaging out of time-dependent factors such as the SD-based variations. Analogously to the 24-hour search, the upper limit on the total emitted energy in terms of neutrinos per source, $E_{\text {up }}$, can be calculated. The resulting value for $E_{\text {up }}$ is approximately $6.3 \cdot 10^{51} \mathrm{erg}$, close to the one for the 24-hour search. Since the longer integration time counters the search duration $T$ in the denominator in Equation 2, the only difference between this $E_{\text {up }}$ and the one for the 24-hour search is the slight variation in the status of the SD. As can be inferred from Figure 4, the long term combined upper limit is on average slightly higher than the very first combined upper limit value in that figure, which corresponds to the first sidereal day after the merger. This translates directly to the slightly less constraining upper limit on the total neutrino emission energy of $6.3 \cdot 10^{51} \mathrm{erg}$ versus $6.0 \cdot 10^{51} \mathrm{erg}$.

\section{Discussion and conclusions}

We have presented a stacking search for UHE neutrinos from LIGO/Virgo BBH mergers with the Pierre Auger Observatory. As no UHE neutrino candidates have been identified in temporal and directional coincidence with any of the BBH mergers, we have obtained a $90 \%$ C.L. upper limit on a universal isotropic UHE neutrino luminosity as a function of time after the merger, $L_{\text {up }}\left(t-t_{0}\right)$, that applies to each source. The temporal coincidence has been probed for two search periods, starting at the time of the merger and lasting for 24 hours and 60 days, respectively. The directional coincidence has been probed by applying all searches to the most probable $90 \%$ quantile of the source's directional localization PDF. The limit is based on the time-dependent visibility of the sources, which is a combination of the direction-dependent instantaneous neutrino exposure of the Pierre Auger Observatory and the sources' 3D localization PDFs. It holds for the assumption of a neutrino emission $\propto E_{v}^{-2}$, which is a common benchmark assumption for UHE neutrino searches.

For the 24-hour search, corresponding to a comparably short emission, the upper limits on the universal isotropic neutrino emission are mostly below $10^{47} \frac{\mathrm{erg}}{\mathrm{s}}$, with moderate variations due to 
the sources' instantaneous visibility. The corresponding upper limit on the total neutrino emission energy per source during the 24 hours after the merger is $6.0 \cdot 10^{51} \mathrm{erg}$. This is a factor of $\sim 2.2$ more constraining than the value corresponding to the previously published search [14], while approximately 3 times as many sources have been followed up. The reason for this worse than linear improvement of the energy limit with the number of sources is the increasing sensitivity of the LIGO and Virgo detectors from run to run, leading to a larger average distance of BBH mergers being reported for the later runs. These more distant BBH mergers contribute less to the luminosity limit than the mergers of the earlier runs, which are on average closer to Earth.

The 60-day search, corresponding to a longer emission duration, leads to overall approximately 60 times smaller values for $L_{\text {up }}\left(t-t_{0}\right)$ than the 24-hour search, as expected from Equation 1, which indicates that the same upper limit on the total number of neutrino induced events, $N_{\text {up }, v}$, is distributed over a longer time period $T$. Another interpretational approach would be an approximately 60 times larger total exposure with the same statistical constraints by zero detected neutrinos and an expected background also very close to zero, which is the reason why the same value of $N_{\text {up, } v}$ applies to both search periods. The combined luminosity limit as a function of sidereal time is similar for every considered sidereal day after the merger as shown in Figure 3. This similarity is a consequence of the periodic course of the Pierre Auger Observatory's field of view. The deviations between the lines in Figure 3 are due to changes in the status of the SD over the course of the 60 days. Figure 4 , which shows the sidereal daily mean values of $L_{\text {up }}\left(t-t_{0}\right)$, illustrates that these deviations are overall small for the combined limit. For O1, an LVC run with only three sources, the deviations over the 60 days are substantial, demonstrating that the combined limit's time stability is another benefit of the source stacking, along with the substantial improvement of the sensitivity itself as compared to single source searches. The upper limit on the total neutrino emission energy per source during the 60 days after the merger is $6.3 \cdot 10^{51} \mathrm{erg}$, which is close to the value for the 24 hour search period because the time integration yielding this limit balances the longer time period $T$ in the denominator in Equation 1. The proximity in the energy upper limits for the two periods further confirms the temporal stability of the stacking search.

As the neutrino emission energy limit is - to first order - independent of the observation duration, it is a robust basis for interpretations and comparisons. It corresponds to approximately $M_{\odot} c^{2} / 300$, whereas the $\mathrm{BBH}$ mergers with published emitted GW energies typically emit a few $M_{\odot} c^{2}[15,17]$. This means that the limit is strong enough to be constraining or at least highly relevant for theoretical considerations such as in [36], keeping in mind the assumptions made for this work, in particular the isotropic emission and the $E_{v}^{-2}$ flux. Another comparison is possible with similar searches performed with IceCube [37], which impose limits in the range of $1.37 \cdot 10^{52} \mathrm{erg}$ through $1.80 \cdot 10^{55} \mathrm{erg}$ to BBH mergers of the GWTC-1 catalogue. A simple combination of the energy upper limits from [37] by adding the inverse of the energy limits, which would be proportional to the exposure, and taking the inverse of the result yields an upper limit on the universal isotropic neutrino emission energy of $E_{\mathrm{up}, \mathrm{IC}} \sim 10^{52} \mathrm{erg}$, whereas the corresponding value for these sources with Auger is $\sim 2.3 \cdot 10^{52} \mathrm{erg}$. As the search by IceCube is focusing on a lower energy range, this result emphasizes the complementarity of the two observatories, while the previously discussed stronger constraint on the universal neutrino emission when considering all sources shows the strength of this stacking analysis method. 


\section{References}

[1] A. Aab et al. [Pierre Auger Coll.], Nucl. Instrum. Meth. A 798 (2015) 172-213, [1502 .01323].

[2] A. Aab et al. [Pierre Auger Coll.], J. Cosmol. Astropart. Phys. 10 (2019) 022, [1906. 07422].

[3] A. Aab et al. [Pierre Auger Coll.], J. Cosmol. Astropart. Phys. 11 (2019) 004, [1906.07419].

[4] K. Greisen, Phys. Rev. Lett. 16 (1966) 748.

[5] G. T. Zatsepin and V. A. Kuzmin, J. Exp. Theor. Phys. Lett. 4 (1966) 78.

[6] D. Hooper, A. Taylor, S. Sarkar, Astropart. Phys. 23 (2005) 11, [astro-ph/0407618].

[7] M. Ave, N. Busca, A. V. Olinto, A. A. Watson, and T. Yamamoto, Astropart. Phys. 23 (2005) 19, [astro-ph/0409316].

[8] K. Kotera, D. Allard, and A. V. Olinto, J. Cosmol. Astropart. Phys. 2010 (2010) 013, [1009.1382].

[9] B. P. Abbot et al. [LIGO Scientific Coll. and Virgo Coll.], Phys. Rev. Lett. 116 (2016) 061102, [1602 . 03837].

[10] B. P. Abbot et al. [LIGO Scientific Coll. and Virgo Coll.], Phys. Rev. Lett. 116 (2016) 241103, [1606.04855].

[11] V. Connaughton et al., Astrophys. J. Lett. 826 (2016) L6, [1602. 03920].

[12] V. Connaughton et al., Astrophys. J. Lett. 853 (2018) L9, [1801. 02305].

[13] M. J. Graham et al., Phys. Rev. Lett. 124 (2020) 251102, [2006.14122].

[14] M. Schimp [for the Pierre Auger Coll.], Proc. 36th Int. Cosmic Ray Conf., Madison, USA (2019), PoS(ICRC2019) 415.

[15] B. P. Abbott et al. [LIGO Scientific Coll. and Virgo Coll.], Phys. Rev. X 9 (2019) 031040, [1811. 12907v3].

[16] A. Albert et al. [ANTARES Coll., IceCube Coll., Pierre Auger Coll., and LIGO Scientific Coll. and Virgo Coll.], Astrophys. J. Lett. 850 (2017) L35, [1710. 05839].

[17] B. P. Abbot et al. [LIGO Scientific Coll. and Virgo Coll.], Phys. Rev. X 11 (2021) 021053, [2010. 14527v3].

[18] B. Piotrzkowski [LIGO Scientific Coll. and Virgo Coll.], GCN Circular 26245 (2019).

[19] B. Piotrzkowski [LIGO Scientific Coll. and Virgo Coll.], GCN Circular 26202 (2019).

[20] L. Singer [LIGO Scientific Coll. and Virgo Coll.], GCN Circular 26383 (2019).

[21] D. Chatterjee [LIGO Scientific Coll. and Virgo Coll.], GCN Circular 26334 (2019).

[22] N. Christensen [LIGO Scientific Coll. and Virgo Coll.], GCN Circular 26518 (2019).

[23] B. Piotrzkowski [LIGO Scientific Coll. and Virgo Coll.], GCN Circular 26570 (2019).

[24] D. Holz [LIGO Scientific Coll. and Virgo Coll.], GCN Circular 26572 (2019).

[25] E. Katsavounidis [LIGO Scientific Coll. and Virgo Coll.], GCN Circular 26715 (2020).

[26] E. Katsavounidis [LIGO Scientific Coll. and Virgo Coll.], GCN Circular 26906 (2020).

[27] B. Piotrzkowski [LIGO Scientific Coll. and Virgo Coll.], GCN Circular 26926 (2020).

[28] L. Singer [LIGO Scientific Coll. and Virgo Coll.], GCN Circular 27036 (2020).

[29] L. Singer [LIGO Scientific Coll. and Virgo Coll.], GCN Circular 27214 (2020).

[30] V. Valsan [LIGO Scientific Coll. and Virgo Coll.], GCN Circular 27262 (2020).

[31] L. Singer [LIGO Scientific Coll. and Virgo Coll.], GCN Circular 27229 (2020).

[32] P. Shawhan [LIGO Scientific Coll. and Virgo Coll.], GCN Circular 27292 (2020).

[33] G. Mo [LIGO Scientific Coll. and Virgo Coll.], GCN Circular 27382 (2020).

[34] M. R. Calabretta and B. F. Roukema, Mon. Not. R. Astron. Soc. 381 (2007) 865, [astro-ph/0412607].

[35] G. J. Feldman and R. D. Cousins, Phys. Rev. D 57 (1998) 3873, [physics/9711021].

[36] K. Kotera and J. Silk, Astrophys. J. Lett. 823 (2016) L29, [1602 . 06961].

[37] M. G. Aartsen et al. [IceCube Coll.], Astrophys. J. Lett. 898 (2020) L10, [2004 . 02910]. 


\section{The Pierre Auger Collaboration}

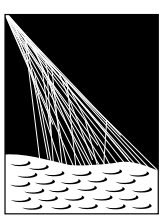

PIERRE

AUSGERVATORY

P. Abreu ${ }^{72}$, M. Aglietta ${ }^{54,52}$, J.M. Albury ${ }^{13}$, I. Allekotte ${ }^{1}$, A. Almela ${ }^{8,12}$, J. Alvarez-Muñiz ${ }^{79}$, R. Alves Batista $^{80}$, G.A. Anastasi ${ }^{63,52}$, L. Anchordoqui ${ }^{87}$, B. Andrada ${ }^{8}$, S. Andringa ${ }^{72}$, C. $\mathrm{Aramo}^{50}$, P.R. Araújo Ferreira ${ }^{42}$, J. C. Arteaga Velázquez ${ }^{67}$, H. Asorey $^{8}$, P. Assis ${ }^{72}$, G. Avila ${ }^{11}$, A.M. Badescu ${ }^{75}$, A. Bakalova ${ }^{32}$, A. Balaceanu ${ }^{73}$, F. Barbato ${ }^{45,46}$, R.J. Barreira Luz $^{72}$, K.H. Becker ${ }^{38}$, J.A. Bellido ${ }^{13,69}$, C. Berat ${ }^{36}$, M.E. Bertaina ${ }^{63,52}$, X. Bertou ${ }^{1}$, P.L. Biermann ${ }^{b}$, V. Binet ${ }^{6}$, K. Bismark ${ }^{39,8}$, T. Bister ${ }^{42}$, J. Biteau ${ }^{37}$, J. Blazek ${ }^{32}$, C. Bleve ${ }^{36}$, M. Boháčová ${ }^{32}$, D. Boncioli ${ }^{57,46}$, C. Bonifazi ${ }^{9,26}$, L. Bonneau Arbeletche ${ }^{21}$, N. Borodai ${ }^{70}$, A.M. Botti ${ }^{8}$, J. Brack ${ }^{d}$, T. Bretz ${ }^{42}$, P.G. Brichetto Orchera ${ }^{8}$, F.L. Briechle ${ }^{42}$, P. Buchholz ${ }^{44}$, A. Bueno ${ }^{78}$, S. Buitink ${ }^{15}$, M. Buscemi ${ }^{47}$, M. Büsken ${ }^{39,8}$, K.S. Caballero-Mora ${ }^{66}$, L. Caccianiga ${ }^{59,49}$, F. Canfora ${ }^{80,81}$, I. Caracas ${ }^{38}$, J.M. Carceller ${ }^{78}$, R. Caruso ${ }^{58,47}$, A. Castellina ${ }^{54,52}$, F. Catalanii ${ }^{19}$, G. Cataldi ${ }^{48}$, L. Cazon ${ }^{72}$, M. Cerda ${ }^{10}$, J.A. Chinellato ${ }^{22}$, J. Chudoba ${ }^{32}$, L. Chytka ${ }^{33}$, R.W. Clay ${ }^{13}$, A.C. Cobos Ceruttii ${ }^{7}$, R. Colalillo ${ }^{60,50}$, A. Coleman ${ }^{93}$, M.R. Coluccia ${ }^{48}$, R. Conceição ${ }^{72}$, A. Condorelli ${ }^{45,46}$, G. Consolati ${ }^{49,55}$, F. Contreras ${ }^{11}$, F. Convenga ${ }^{56,48}$, D. Correia dos Santos $^{28}$, C.E. Covault ${ }^{85}$, S. Dasso ${ }^{5,3}$, K. Daumiller ${ }^{41}$, B.R. Dawson ${ }^{13}$, J.A. Day ${ }^{13}$, R.M. de Almeida ${ }^{28}$, J. de Jesús ${ }^{8,41}$, S.J. de Jong ${ }^{80,81}$, G. De Mauro ${ }^{80,81}$, J.R.T. de Mello Neto ${ }^{26,27}$, I. De Mitri ${ }^{45,46}$, J. de Oliveira ${ }^{18}$, D. de Oliveira Franco ${ }^{22}$, F. de Palma ${ }^{56,48}$, V. de Souza $^{20}$, E. De Vito ${ }^{56,48}$, M. del Río ${ }^{11}$, O. Deligny ${ }^{34}$, L. Deval ${ }^{41,8}$, A. di Matteo $^{52}$, C. Dobrigkeit ${ }^{22}$, J.C. D’Olivo ${ }^{68}$, L.M. Domingues Mendes ${ }^{72}$, R.C. dos Anjos ${ }^{25}$, D. dos Santos $^{28}$, M.T. Dova ${ }^{4}$, J. Ebr ${ }^{32}$, R. Engel ${ }^{39,41}$, I. Epicoco ${ }^{56,48}$, M. Erdmann ${ }^{42}$, C.O. Escobar ${ }^{a}$, A. Etchegoyen ${ }^{8,12}$, H. Falcke ${ }^{80,82,81}$, J. Farmer ${ }^{92}$, G. Farrar ${ }^{90}$, A.C. Fauth ${ }^{22}$, N. Fazzini ${ }^{a}$, F. Feldbusch ${ }^{40}$, F. Fenu ${ }^{54,52}$,

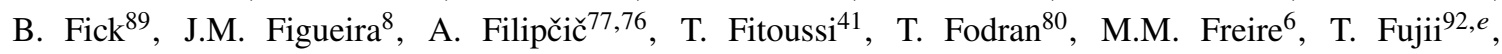
A. Fuster $^{8,12}$, C. Galea ${ }^{80}$, C. Galelli ${ }^{59,49}$, B. García ${ }^{7}$, A.L. Garcia Vegas ${ }^{42}$, H. Gemmeke ${ }^{40}$, F. Gesualdi ${ }^{8,41}$, A. Gherghel-Lascu ${ }^{73}$, P.L. Ghia ${ }^{34}$, U. Giaccari ${ }^{80}$, M. Giammarchi ${ }^{49}$, J. Glombitza ${ }^{42}$, F. Gobbi ${ }^{10}$, F. Gollan ${ }^{8}$, G. Golup ${ }^{1}$, M. Gómez Berisso ${ }^{1}$, P.F. Gómez Vitale ${ }^{11}$, J.P. Gongora ${ }^{11}$, J.M. González ${ }^{1}$, N. González ${ }^{14}$, I. Goos ${ }^{1,41}$, D. Góra ${ }^{70}$, A. Gorgi ${ }^{54,52}$, M. Gottowik ${ }^{38}$, T.D. Grubb ${ }^{13}$, F. Guarino ${ }^{60,50}$, G.P. Guedes ${ }^{23}$, E. Guido ${ }^{52,63}$, S. $\mathrm{Hahn}^{41,8}$, P. $\mathrm{Hamal}^{32}$, M.R. Hampel ${ }^{8}$, P. Hansen ${ }^{4}$, D. Harari ${ }^{1}$, V.M. Harvey ${ }^{13}$, A. Haungs ${ }^{41}$, T. Hebbeker ${ }^{42}$, D. Heck ${ }^{41}$, G.C. Hill ${ }^{13}$, C. Hojvat ${ }^{a}$, J.R. Hörandel ${ }^{80,81}$, P. Horvath ${ }^{33}$, M. Hrabovský ${ }^{33}$, T. Huege ${ }^{41,15}$, A. Insolia ${ }^{58,47}$, P.G. Isar $^{74}$, P. Janecek ${ }^{32}$, J.A. Johnsen ${ }^{86}$, J. Jurysek ${ }^{32}$, A. Kääpä ${ }^{38}$, K.H. Kampert ${ }^{38}$, N. Karastathis ${ }^{41}$, B. Keilhauer ${ }^{41}$, J. Kemp ${ }^{42}$, A. Khakurdikar ${ }^{80}$, V.V. Kizakke Covilakam $^{8,41}$, H.O. Klages ${ }^{41}$, M. Kleifges ${ }^{40}$, J. Kleinfeller ${ }^{10}$, M. Köpke ${ }^{39}$, N. Kunka ${ }^{40}$, B.L. Lago ${ }^{17}$, R.G. Lang ${ }^{20}$, N. Langner ${ }^{42}$, M.A. Leigui de Oliveira ${ }^{24}$, V. Lenok $^{41}$, A. Letessier-Selvon ${ }^{35}$, I. LhenryYvon $^{34}$, D. Lo Presti ${ }^{58,47}$, L. Lopes ${ }^{72}$, R. López ${ }^{64}$, L. Lu ${ }^{94}$, Q. Luce ${ }^{39}$, J.P. Lundquist ${ }^{76}$, A. Machado Payeras $^{22}$, G. Mancarella ${ }^{56,48}$, D. Mandat ${ }^{32}$, B.C. Manning ${ }^{13}$, J. Manshanden ${ }^{43}$, P. Mantsch ${ }^{a}$, S. Marafico ${ }^{34}$, A.G. Mariazzi ${ }^{4}$, I.C. Mariş ${ }^{14}$, G. Marsella ${ }^{61,47}$, D. Martello ${ }^{56,48}$, S. Martinelli ${ }^{41,8}$, O. Martínez Bravo ${ }^{64}$, M. Mastrodicasa ${ }^{57,46}$, H.J. Mathes ${ }^{41}$, J. Matthews ${ }^{88}$, G. Matthiae ${ }^{62,51}$, E. Mayotte ${ }^{38}$, P.O. Mazur ${ }^{a}$, G. MedinaTanco $^{68}$, D. Melo $^{8}$, A. Menshikov ${ }^{40}$, K.-D. Merenda ${ }^{86}$, S. Michal ${ }^{33}$, M.I. Micheletti ${ }^{6}$, L. Miramonti ${ }^{59,49}$, S. Mollerach ${ }^{1}$, F. Montanet ${ }^{36}$, C. Morello ${ }^{54,52}$, M. Mostafá ${ }^{91}$, A.L. Müller ${ }^{8}$, M.A. Muller ${ }^{22}$, K. Mulrey ${ }^{15}$, R. Mussa ${ }^{52}$, M. Muzio ${ }^{90}$, W.M. Namasaka ${ }^{38}$, A. Nasr-Esfahani ${ }^{38}$, L. Nellen ${ }^{68}$, M. Niculescu-Oglinzanu ${ }^{73}$, M. Niechciol ${ }^{44}$, D. Nitz ${ }^{89}$, D. Nosek ${ }^{31}$, V. Novotny ${ }^{31}$, L. Nožka ${ }^{33}$, A Nucita ${ }^{56,48}$, L.A. Núñez ${ }^{30}$, M. Palatka ${ }^{32}$, J. Pallotta ${ }^{2}$, P. Papenbreer ${ }^{38}$, G. Parente ${ }^{79}$, A. Parra ${ }^{64}$, J. Pawlowsky ${ }^{38}$, M. Pech ${ }^{32}$, F. Pedreira ${ }^{79}$, J. Pȩkala ${ }^{70}$, R. Pelayo ${ }^{65}$, J. Peña-Rodriguez ${ }^{30}$, E.E. Pereira Martins ${ }^{39,8}$, J. Perez Armand ${ }^{21}$, C. Pérez Bertolli $^{8,41}$, M. Perlin ${ }^{8,41}$, L. Perrone ${ }^{56,48}$, S. Petrera ${ }^{45,46}$, T. Pierog ${ }^{41}$, M. Pimenta ${ }^{72}$, V. Pirronello ${ }^{58,47}$, M. Platino ${ }^{8}$, B. Pont $^{80}$, M. Pothast ${ }^{81,80}$, P. Privitera ${ }^{92}$, M. Prouza ${ }^{32}$, A. Puyleart ${ }^{89}$, S. Querchfeld ${ }^{38}$, J. Rautenberg ${ }^{38}$, D. Ravignani ${ }^{8}$, M. Reininghaus ${ }^{41,8}$, J. Ridky ${ }^{32}$, F. Riehn ${ }^{72}$, M. Risse ${ }^{44}$, V. Rizi ${ }^{57,46}$, W. Rodrigues de Carvalho ${ }^{21}$, J. Rodriguez Rojo ${ }^{11}$, M.J. Roncoroni ${ }^{8}$, S. Rossoni ${ }^{43}$, M. Roth ${ }^{41}$, E. Roulet ${ }^{1}$, A.C. Rovero ${ }^{5}$, P. Ruehl ${ }^{44}$, A. Saftoiu ${ }^{73}$, F. Salamida ${ }^{57,46}$, H. Salazar ${ }^{64}$, G. Salina ${ }^{51}$, J.D. Sanabria Gomez ${ }^{30}$, F. Sánchez ${ }^{8}$, E.M. Santos ${ }^{21}$, E. Santos ${ }^{32}$, F. Sarazin ${ }^{86}$, R. Sarmento ${ }^{72}$, C. Sarmiento-Cano ${ }^{8}$, R. Sato ${ }^{11}$, 
P. Savina ${ }^{56,48,34,94}$, C.M. Schäfer ${ }^{41}$, V. Scherini ${ }^{56,48}$, H. Schieler ${ }^{41}$, M. Schimassek ${ }^{39,8}$, M. Schimp ${ }^{38}$, F. Schlüter ${ }^{41,8}$, D. Schmidt ${ }^{39}$, O. Scholten ${ }^{84,15}$, P. Schovánek ${ }^{32}$, F.G. Schröder ${ }^{93,41}$, S. Schröder ${ }^{38}$, J. Schulte ${ }^{42}$, S.J. Sciutto ${ }^{4}$, M. Scornavacche ${ }^{8,41}$, A. Segreto ${ }^{53,47}$, S. Sehgal ${ }^{38}$, R.C. Shellard ${ }^{16}$, G. Sigl ${ }^{43}$, G. Silli ${ }^{8,41}$, O. Sima ${ }^{73, f}$, R. Šmída ${ }^{92}$, P. Sommers ${ }^{91}$, J.F. Soriano ${ }^{87}$, J. Souchard ${ }^{36}$, R. Squartini ${ }^{10}$, M. Stadelmaier ${ }^{41,8}$, D. Stanca ${ }^{73}$, S. Staničc ${ }^{76}$, J. Stasielak ${ }^{70}$, P. Stassi ${ }^{36}$, A. Streich ${ }^{39,8}$, M. Suárez-Durán ${ }^{14}$, T. Sudholz ${ }^{13}$, T. Suomijärvi ${ }^{37}$, A.D. Supanitsky ${ }^{8}$, Z. Szadkowski ${ }^{71}$, A. Tapia ${ }^{29}$, C. Taricco ${ }^{63,52}$, C. Timmermans ${ }^{81,80}$, O. Tkachenko ${ }^{41}$, P. Tobiska ${ }^{32}$, C.J. Todero Peixoto ${ }^{19}$, B. Tomé ${ }^{72}$, Z. Torrès ${ }^{36}$, A. Travaini ${ }^{10}$, P. Travnicek ${ }^{32}$, C. Trimarelli ${ }^{57,46}$, M. Tueros ${ }^{4}$, R. Ulrich ${ }^{41}$, M. Unger ${ }^{41}$, L. Vaclavek ${ }^{33}$, M. Vacula ${ }^{33}$, J.F. Valdés Galicia ${ }^{68}$, L. Valore ${ }^{60,50}$, E. Varela ${ }^{64}$, A. Vásquez-Ramírez ${ }^{30}$, D. Veberič ${ }^{41}$, C. Ventura ${ }^{27}$, I.D. Vergara Quispe ${ }^{4}$, V. Verzi ${ }^{51}$, J. Vicha ${ }^{32}$, J. Vink ${ }^{83}$, S. Vorobiov ${ }^{76}$, H. Wahlberg ${ }^{4}$, C. Watanabe ${ }^{26}$, A.A. Watson ${ }^{c}$, M. Weber ${ }^{40}$, A. Weindl ${ }^{41}$, L. Wiencke ${ }^{86}$, H. Wilczyński ${ }^{70}$, M. Wirtz ${ }^{42}$, D. Wittkowski ${ }^{38}$, B. Wundheiler ${ }^{8}$, A. Yushkov $^{32}$, O. Zapparrata ${ }^{14}$, E. Zas ${ }^{79}$, D. Zavrtanik ${ }^{76,77}$, M. Zavrtanik ${ }^{77,76}$, L. Zehrer ${ }^{76}$

${ }^{1}$ Centro Atómico Bariloche and Instituto Balseiro (CNEA-UNCuyo-CONICET), San Carlos de Bariloche, Argentina

${ }^{2}$ Centro de Investigaciones en Láseres y Aplicaciones, CITEDEF and CONICET, Villa Martelli, Argentina

${ }^{3}$ Departamento de Física and Departamento de Ciencias de la Atmósfera y los Océanos, FCEyN, Universidad de Buenos Aires and CONICET, Buenos Aires, Argentina

${ }^{4}$ IFLP, Universidad Nacional de La Plata and CONICET, La Plata, Argentina

${ }^{5}$ Instituto de Astronomía y Física del Espacio (IAFE, CONICET-UBA), Buenos Aires, Argentina

${ }^{6}$ Instituto de Física de Rosario (IFIR) - CONICET/U.N.R. and Facultad de Ciencias Bioquímicas y Farmacéuticas U.N.R., Rosario, Argentina

${ }^{7}$ Instituto de Tecnologías en Detección y Astropartículas (CNEA, CONICET, UNSAM), and Universidad Tecnológica Nacional - Facultad Regional Mendoza (CONICET/CNEA), Mendoza, Argentina

${ }^{8}$ Instituto de Tecnologías en Detección y Astropartículas (CNEA, CONICET, UNSAM), Buenos Aires, Argentina

${ }^{9}$ International Center of Advanced Studies and Instituto de Ciencias Físicas, ECyT-UNSAM and CONICET, Campus Miguelete - San Martín, Buenos Aires, Argentina

${ }^{10}$ Observatorio Pierre Auger, Malargüe, Argentina

${ }^{11}$ Observatorio Pierre Auger and Comisión Nacional de Energía Atómica, Malargüe, Argentina

12 Universidad Tecnológica Nacional - Facultad Regional Buenos Aires, Buenos Aires, Argentina

${ }^{13}$ University of Adelaide, Adelaide, S.A., Australia

14 Université Libre de Bruxelles (ULB), Brussels, Belgium

15 Vrije Universiteit Brussels, Brussels, Belgium

${ }^{16}$ Centro Brasileiro de Pesquisas Fisicas, Rio de Janeiro, RJ, Brazil

${ }^{17}$ Centro Federal de Educação Tecnológica Celso Suckow da Fonseca, Nova Friburgo, Brazil

${ }^{18}$ Instituto Federal de Educação, Ciência e Tecnologia do Rio de Janeiro (IFRJ), Brazil

${ }^{19}$ Universidade de São Paulo, Escola de Engenharia de Lorena, Lorena, SP, Brazil

${ }^{20}$ Universidade de São Paulo, Instituto de Física de São Carlos, São Carlos, SP, Brazil

${ }^{21}$ Universidade de São Paulo, Instituto de Física, São Paulo, SP, Brazil

${ }^{22}$ Universidade Estadual de Campinas, IFGW, Campinas, SP, Brazil

${ }^{23}$ Universidade Estadual de Feira de Santana, Feira de Santana, Brazil

${ }^{24}$ Universidade Federal do ABC, Santo André, SP, Brazil

${ }^{25}$ Universidade Federal do Paraná, Setor Palotina, Palotina, Brazil

${ }^{26}$ Universidade Federal do Rio de Janeiro, Instituto de Física, Rio de Janeiro, RJ, Brazil

${ }^{27}$ Universidade Federal do Rio de Janeiro (UFRJ), Observatório do Valongo, Rio de Janeiro, RJ, Brazil

${ }^{28}$ Universidade Federal Fluminense, EEIMVR, Volta Redonda, RJ, Brazil

${ }^{29}$ Universidad de Medellín, Medellín, Colombia

${ }^{30}$ Universidad Industrial de Santander, Bucaramanga, Colombia

${ }^{31}$ Charles University, Faculty of Mathematics and Physics, Institute of Particle and Nuclear Physics, Prague, Czech Republic

32 Institute of Physics of the Czech Academy of Sciences, Prague, Czech Republic 
${ }^{33}$ Palacky University, RCPTM, Olomouc, Czech Republic

34 CNRS/IN2P3, IJCLab, Université Paris-Saclay, Orsay, France

${ }^{35}$ Laboratoire de Physique Nucléaire et de Hautes Energies (LPNHE), Sorbonne Université, Université de Paris, CNRSIN2P3, Paris, France

${ }^{36}$ Univ. Grenoble Alpes, CNRS, Grenoble Institute of Engineering Univ. Grenoble Alpes, LPSC-IN2P3, 38000 Grenoble, France

${ }^{37}$ Université Paris-Saclay, CNRS/IN2P3, IJCLab, Orsay, France

38 Bergische Universität Wuppertal, Department of Physics, Wuppertal, Germany

${ }^{39}$ Karlsruhe Institute of Technology (KIT), Institute for Experimental Particle Physics, Karlsruhe, Germany

${ }^{40}$ Karlsruhe Institute of Technology (KIT), Institut für Prozessdatenverarbeitung und Elektronik, Karlsruhe, Germany

${ }^{41}$ Karlsruhe Institute of Technology (KIT), Institute for Astroparticle Physics, Karlsruhe, Germany

${ }^{42}$ RWTH Aachen University, III. Physikalisches Institut A, Aachen, Germany

${ }^{43}$ Universität Hamburg, II. Institut für Theoretische Physik, Hamburg, Germany

${ }^{44}$ Universität Siegen, Department Physik - Experimentelle Teilchenphysik, Siegen, Germany

${ }^{45}$ Gran Sasso Science Institute, L'Aquila, Italy

46 INFN Laboratori Nazionali del Gran Sasso, Assergi (L'Aquila), Italy

${ }^{47}$ INFN, Sezione di Catania, Catania, Italy

${ }^{48}$ INFN, Sezione di Lecce, Lecce, Italy

${ }^{49}$ INFN, Sezione di Milano, Milano, Italy

${ }^{50}$ INFN, Sezione di Napoli, Napoli, Italy

${ }^{51}$ INFN, Sezione di Roma "Tor Vergata", Roma, Italy

52 INFN, Sezione di Torino, Torino, Italy

53 Istituto di Astrofisica Spaziale e Fisica Cosmica di Palermo (INAF), Palermo, Italy

54 Osservatorio Astrofisico di Torino (INAF), Torino, Italy

55 Politecnico di Milano, Dipartimento di Scienze e Tecnologie Aerospaziali, Milano, Italy

${ }^{56}$ Università del Salento, Dipartimento di Matematica e Fisica "E. De Giorgi”, Lecce, Italy

57 Università dell'Aquila, Dipartimento di Scienze Fisiche e Chimiche, L'Aquila, Italy

58 Università di Catania, Dipartimento di Fisica e Astronomia, Catania, Italy

${ }^{59}$ Università di Milano, Dipartimento di Fisica, Milano, Italy

${ }^{60}$ Università di Napoli "Federico II", Dipartimento di Fisica "Ettore Pancini”, Napoli, Italy

${ }^{61}$ Università di Palermo, Dipartimento di Fisica e Chimica "E. Segrè", Palermo, Italy

62 Università di Roma "Tor Vergata", Dipartimento di Fisica, Roma, Italy

${ }^{63}$ Università Torino, Dipartimento di Fisica, Torino, Italy

${ }^{64}$ Benemérita Universidad Autónoma de Puebla, Puebla, México

${ }^{65}$ Unidad Profesional Interdisciplinaria en Ingeniería y Tecnologías Avanzadas del Instituto Politécnico Nacional (UPIITA-IPN), México, D.F., México

66 Universidad Autónoma de Chiapas, Tuxtla Gutiérrez, Chiapas, México

${ }^{67}$ Universidad Michoacana de San Nicolás de Hidalgo, Morelia, Michoacán, México

${ }^{68}$ Universidad Nacional Autónoma de México, México, D.F., México

${ }^{69}$ Universidad Nacional de San Agustin de Arequipa, Facultad de Ciencias Naturales y Formales, Arequipa, Peru

${ }^{70}$ Institute of Nuclear Physics PAN, Krakow, Poland

${ }^{71}$ University of Łódź, Faculty of High-Energy Astrophysics, Łódź, Poland

${ }^{72}$ Laboratório de Instrumentação e Física Experimental de Partículas - LIP and Instituto Superior Técnico - IST, Universidade de Lisboa - UL, Lisboa, Portugal

73 "Horia Hulubei” National Institute for Physics and Nuclear Engineering, Bucharest-Magurele, Romania

${ }^{74}$ Institute of Space Science, Bucharest-Magurele, Romania

75 University Politehnica of Bucharest, Bucharest, Romania

76 Center for Astrophysics and Cosmology (CAC), University of Nova Gorica, Nova Gorica, Slovenia

${ }^{77}$ Experimental Particle Physics Department, J. Stefan Institute, Ljubljana, Slovenia

78 Universidad de Granada and C.A.F.P.E., Granada, Spain

${ }^{79}$ Instituto Galego de Física de Altas Enerxías (IGFAE), Universidade de Santiago de Compostela, Santiago de Compostela, Spain 
${ }^{80}$ IMAPP, Radboud University Nijmegen, Nijmegen, The Netherlands

${ }^{81}$ Nationaal Instituut voor Kernfysica en Hoge Energie Fysica (NIKHEF), Science Park, Amsterdam, The Netherlands

82 Stichting Astronomisch Onderzoek in Nederland (ASTRON), Dwingeloo, The Netherlands

${ }^{83}$ Universiteit van Amsterdam, Faculty of Science, Amsterdam, The Netherlands

${ }^{84}$ University of Groningen, Kapteyn Astronomical Institute, Groningen, The Netherlands

85 Case Western Reserve University, Cleveland, OH, USA

86 Colorado School of Mines, Golden, CO, USA

${ }^{87}$ Department of Physics and Astronomy, Lehman College, City University of New York, Bronx, NY, USA

${ }^{88}$ Louisiana State University, Baton Rouge, LA, USA

${ }^{89}$ Michigan Technological University, Houghton, MI, USA

${ }^{90}$ New York University, New York, NY, USA

${ }^{91}$ Pennsylvania State University, University Park, PA, USA

92 University of Chicago, Enrico Fermi Institute, Chicago, IL, USA

93 University of Delaware, Department of Physics and Astronomy, Bartol Research Institute, Newark, DE, USA

94 University of Wisconsin-Madison, Department of Physics and WIPAC, Madison, WI, USA

${ }^{a}$ Fermi National Accelerator Laboratory, Fermilab, Batavia, IL, USA

${ }^{b}$ Max-Planck-Institut für Radioastronomie, Bonn, Germany

${ }^{c}$ School of Physics and Astronomy, University of Leeds, Leeds, United Kingdom

${ }^{d}$ Colorado State University, Fort Collins, CO, USA

$e^{e}$ now at Hakubi Center for Advanced Research and Graduate School of Science, Kyoto University, Kyoto, Japan

$f$ also at University of Bucharest, Physics Department, Bucharest, Romania 